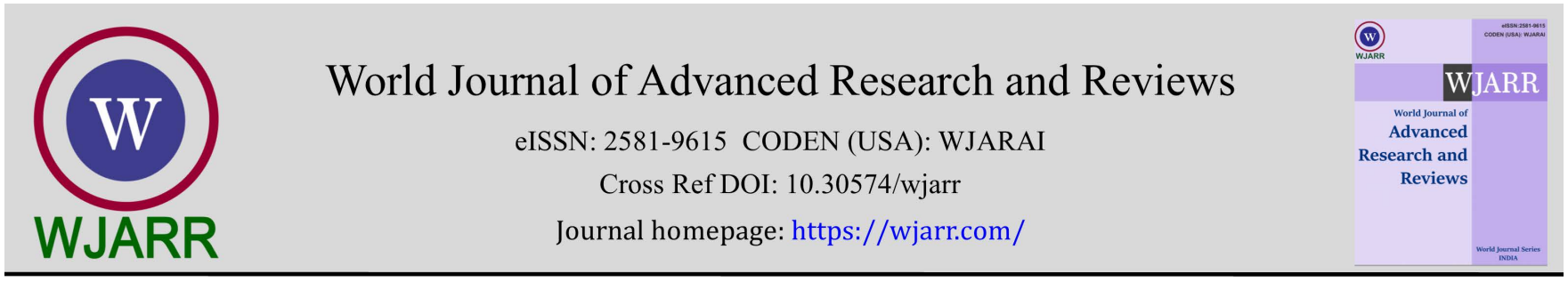

(CASE REPORT)

Check for updates

\title{
Atypical Euglycemic Ketoacidosis in a non-diabetic Patient due to acute pancreatitis
}

\author{
Andree Emanuilov Manov ${ }^{1, *}$ and Ikechukwu Ogbu ${ }^{2}$ \\ ${ }^{1}$ Department of Medicine, Internal Medicine Residency Program, Sunrise Health Consortium GME, Mountain View Hospital, \\ Las Vegas, Nevada, USA.
}

2 Touro University medical College, Henderson, Nevada, USA.

World Journal of Advanced Research and Reviews, 2021, 11(03), 332-336

Publication history: Received on 18 August 2021; revised on 23 September 2021; accepted on 25 September 2021

Article DOI: https://doi.org/10.30574/wjarr.2021.11.3.0461

\begin{abstract}
We describe the case of a previously healthy 40- year-old Female with a known medical history of essential hypertension (HTN). She did not have past medical history of Diabetes Mellitus. She presents to the emergency department with sudden onset of severe shortness of breath that began shortly after non-bilious, non-bloating emesis. She also reported low-grade fever, nausea, cough, abdominal pain, pleuritic chest pain, and generalized weakness. She was found to have acute pancreatitis.
\end{abstract}

She was diaphoretic. She had Kussmaul breathing. She was subsequently admitted to the intensive care unit (ICU) for severe metabolic acidosis. The cause of her metabolic acidosis and clinical presentation was found to be the acute pancreatitis which very rarely can be the cause of euglycemic ketoacidosis.

Keywords: Ketoacidosis 1; Insulin 2; Glucagon 3; Acute pancreatitis 4; Diabetes Mellitus 5; Lipase 6

\section{Introduction}

The patient we are describing was not a known diabetic and her blood glucose was in normal limits. Her HbA1c was normal at 5.2\%. The ketoacidosis we are describing cannot be attributed to Diabetes Mellitus [1-2]. It cannot be attributed to starvation as our patient was not fasting when she was admitted. Furthermore the starvation is very infrequent cause of ketoacidosis. Also the ketoacidosis cannot be attributed to the alcohol. Her alcohol screen of the urine was negative. The ketoacidosis cannot be attributed to vomiting, because she had only couple of episodes to vomiting. Our patient did not have any liver or kidney problems.

In the report in the literature, Kabadi UM described three patients Who manifested ketoacidosis at the time of presentation of acute pancreatitis [3].

In none of these the ketoacidosis could be attributed to any known pathogenetic factor such as alcohol, diabetes mellitus or prolonged starvation. He believed that pancreatic ketoacidosis can be a distinct syndrome with ketogenesis being promoted by high levels of lipase concentration. This was thereafter named "Kabadi syndrome" [3-4]. Our case highlights the diagnostic challenge and the need to be considered this differential diagnosis in patients presenting with metabolic acidosis in the absence of hyperglycemia. Clinicians should also consider the possibility of acute pancreatitis as the underlying illness.

\footnotetext{
* Corresponding author: Andree Emanuilov Manov

Department of Medicine, Internal Medicine Residency Program, Sunrise Health Consortium GME, Mountain View Hospital, Las Vegas, Nevada, USA.

Copyright (C) 2021 Author(s) retain the copyright of this article. This article is published under the terms of the Creative Commons Attribution Liscense 4.0.
} 


\section{Case Presentation}

The patient was a 40-year-old female with a Past Medical History significant for Hypertension that was brought to the emergency department with sudden onset of severe shortness of breath that began shortly after vomiting 2-3 times. Associated symptoms included fever, nausea, cough, abdominal pain, rib pain, pleuritic chest pain, and generalized weakness. Initial vital signs in the Emergency Department were: blood pressure of 156/94, heart rate of 150, Respiratory rate of 44 , oxygen saturation $100 \%$ on $2.5 \mathrm{~L}$ nasal cannula, and temperature of $98.1 \mathrm{~F}$. She was awake and alert but was noted to have Kussmaul breathing and diaphoresis. Other remarkable finding included dry mucus membrane, tachycardia, tachypnea, rib pain, and epigastric tenderness.

Initial laboratory results shown in [Table 1] revealed metabolic acidosis with incomplete respiratory compensation. ABG result showed pH 7.083, PCO2 20, HCO3- 5, PO2 98.8. Complete blood count showed WBC of 8 k/ul with Neutrophil count of $92.29 \%$.

The comprehensive metabolic panel showed Glucose of 123, potassium of 6.4, Anion Gap of 36, Albumin 3.4, AST of 123 units/L, Alkaline Phosphatase of 133 units/L, ALT of 70 units/L. Lipase of 895 unit/L, Serum osmolality of 318 $\mathrm{mOsm} / \mathrm{kg}$, Beta-Hydroxybutyrate Acid $>4.0 \mathrm{mmol} / \mathrm{L}, \mathrm{HbA1c} 5.2 \%$. Urinalysis reveals ketone, Blood alcohol level was negative, and drug screen was negative. ECG shows sinus tachycardia and CTA lung was negative for pulmonary embolism.

Due to the nature of her presentation and elevated liver function test, she was taken to radiology for CXR and CT abdomen and pelvis [Figure 1]. CT of the abdomen revealed changes consistent with acute pancreatitis and there were no gall stones detected. CXR was normal. This presentation was considered an atypical case of increased anion gab metabolic ketoacidosis because of the absence of hyperglycemia and Diabetes Mellitus- HbA1C- 5.2\% and no increased blood sugar during the hospital stay. Because of her High anion gap metabolic acidosis due to acute pancreatitis she was admitted to the medical ICU for further management.

The patient was treated for acute pancreatitis and the ketoacidosis with bowel rest and intravenous fluid- D5 NS at rate $150 \mathrm{ml}$ per hour and intravenous insulin infusion. She had complete resolution of her acidosis and electrolyte abnormalities. She made a full recovery on the medicine floor and was subsequently discharged home.

Table 1 Summary of Laboratory results

\begin{tabular}{|l|c|c|}
\hline Laboratory test (Units) & Initial Values & Values after 13 days \\
\hline Plasma blood glucose (mg/Dl) & 123 & 110 \\
\hline Hemoglobin A1c (\%) & 5.2 & 135 \\
\hline Sodium (mmol/L) & 142 & 4.3 \\
\hline Potassium (mmol/L) & 6.4 & 98 \\
\hline Chloride (mmol/L) & 101 & 11 \\
\hline Anion gap & 36 & 18 \\
\hline Blood urea nitrogen (mg/dL) & 12 & 0.90 \\
\hline Creatinine (mg/dL) & 1.3 & 26 \\
\hline Serum Carbon dioxide (mmol/L) & $<5$ & 3.5 \\
\hline Albumin (g/dL) & 3.4 & \\
\hline Betahydroxylbuterate (mmol/L) & $>4.0$ & 33 \\
\hline Triglyceride (mg/dL) & 112 & 53 \\
\hline AST & 123 & 40 \\
\hline Alkaline Phosphatase & 133 & 70 \\
\hline ALT & & \\
\hline
\end{tabular}




\begin{tabular}{|c|c|c|}
\hline $\mathrm{pH}$ & 7.08 & 7.41 \\
\hline PCO2 (mm Hg) & 20 & 36 \\
\hline PO2 (mm Hg) & 98 & 96 \\
\hline Bicarbonate (mmol/L) & 18 & 26 \\
\hline Urine Ketones & $>160$ & \\
\hline White blood cells (cell $/ \mathrm{mm}^{3}$ ) & 8.0 & 9.5 \\
\hline Hemoglobin (gm/dL) & 16 & 13 \\
\hline Platelet (cell/mm³) & 226 & 211 \\
\hline
\end{tabular}
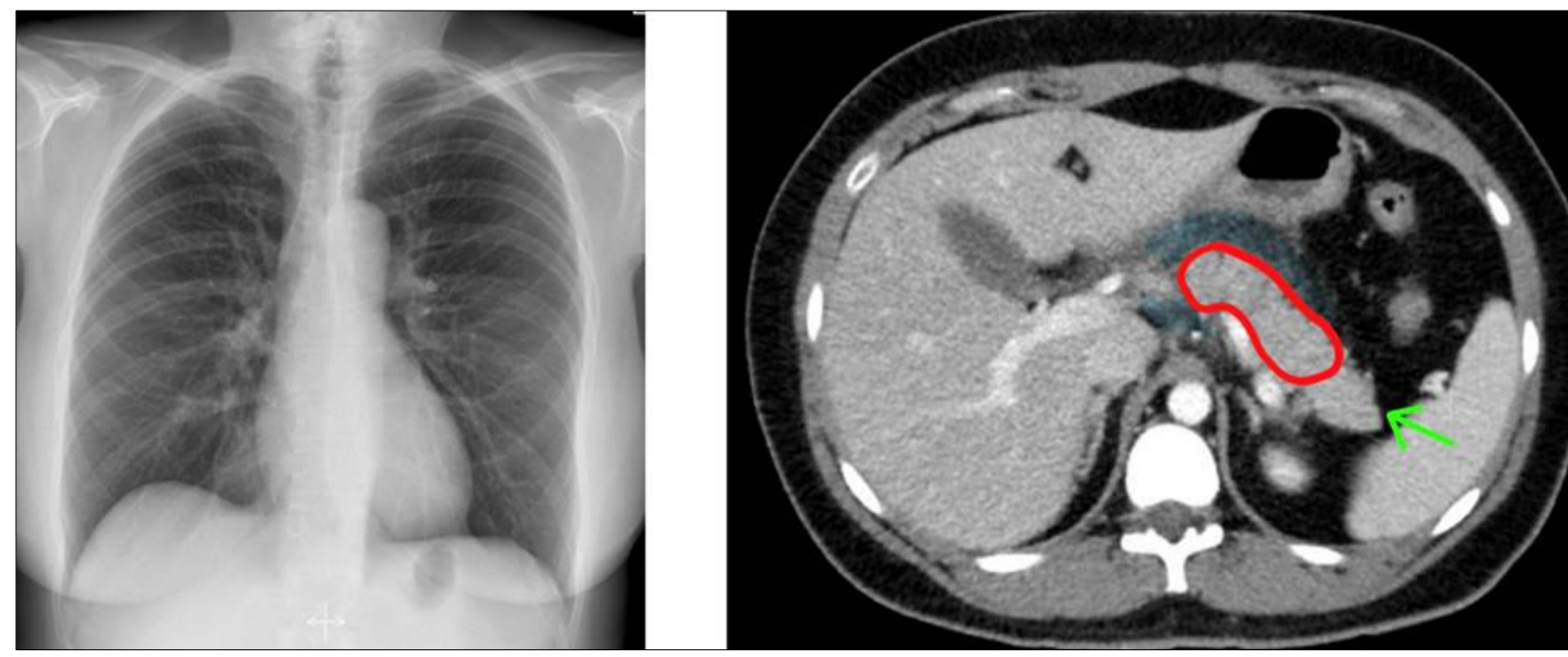

Figure 1 Chest x-ray film (left) and Abdomen computed tomography (right) showing acute pancreatitis

\section{Discussion}

Diabetic Ketoacidosis/DKA/ diagnostic criteria include a combination of hyperglycemia, increased anion gap metabolic acidosis, and ketonuria [1]. DKA usually occurs because of absolute or relative insulin deficiency that is accompanied by an increase in counter-regulatory hormones (i.e., glucagon, cortisol, growth hormone, and epinephrine. Euglycemic DKA/EDKA/ is also thought to be due to starvation and food restriction and inhibition of gluconeogenesis or certain drugs like SGLT2 seen mainly in patients with a history of diabetes and insulin deficiency $[1,2]$.

Our patient in this case report did not have diabetes, her HbA1c was $5.2 \%$ and was she was euglycemic throughout her hospital stay. We do not believe she had euglycemic diabetic ketoacidosis.

This patient described in our case report was diagnosed with atypical euglycemic ketoacidosis /AEKA/due to acute pancreatitis.

Ketonemia and ketoacidosis is well documented as a consequence of prolonged starvation, acute alcoholism and uncontrolled diabetes mellitus. Atypical euglycemic ketoacidosis due to acute pancreatitis is a very rare entity and only few case reports have been reported worldwide.

One known case was reported by Prater and Chaiban, a case of euglycemic ketoacidosis not known to have diabetes, during an acute admission for pancreatitis, suggesting that the insult to the pancreas may have caused hormonal dysregulation [5]. Also another report of atypical euglycemic ketoacidosis due to acute pancreatitis was reported by Kabadi described as discussed above the case of pancreatic ketoacidosis- ketonemia associated with acute pancreatitis known as Kabadi syndrome as well as by Dhivahar [3-4-6]. 
High index of suspicion of atypical euglycemic ketoacidosis is warranted by the clinicians.

Ketosis and ketoacidosis secondary due to acute pancreatitis is a distinct syndrome, induced and maintained by high levels of pancreatic lipase in the circulation. Some studies suggest that the glucagon, the hormone which promotes ketogenesis is nine time higher in first episode of acute pancreatitis and contributes to the atypical euglycemic ketoacidosis [7]. Also a contributor is relative hypoinsulinemia in acute pancreatitis [8]. As per Pillai and Modi pancreatic lipase is responsible for induction and maintaining of ketoacidosis via lipolysis and creation of ketone bodies which can be due to acute pancreatitis and not due to euglycemic DKA [9-10].

The management of atypical euglycemic ketoacidosis is similar to the management of DKA. Initial fluid therapy is directed toward expansion of the intravascular, interstitial, and intracellular volume and restoration of adequate renal perfusion [9-10]. Hence, the mainstay of treatment involves rapid correction of dehydration using intravenous fluids. The second most important step in the management is the use of insulin drip along with a dextrose-containing solution until the anion gap, and bicarbonate levels normalize [6-9-10].

EDKA is a diagnosis of exclusion [11-12-13]. Other forms of ketoacidosis like starvation ketoacidosis should be ruled out. Also, other causes of increased anion gap metabolic acidosis like lactic acidosis, increased toxic serum alcohols (methanol, ethylene glycol, etc.), drug toxicities, paraldehyde ingestion, and renal failure must be excluded. While Infections such as urinary tract infection (UTI) and pneumonia are the most common precipitating factors for DKA, acute Pancreatitis is also a known precipitating factor for the development of DKA and early suspicion for pancreatic disease in Nondiabetic Patients with clinical signs suggestive of DKA, can significantly impact diagnostic and clinical outcomes [2-14]. Successful evaluation and treatment of DKA require frequent monitoring of patients, correction of hypovolemia, electrolyte abnormalities, and hyperglycemia.

\section{Conclusion}

Atypical euglycemic Ketoacidosis possesses a challenge for physicians, as patients presenting with normal Blood glucose levels and ketoacidosis may be overlooked, leading to a delay in appropriate diagnosis and management. Despite euglycemia, ketoacidosis in patients remains a medical emergency and must be treated in a quick and appropriate manner. The rare cases of pancreatitis should be suspected as a cause of this rare type of ketoacidosis in appropriate clinical scenario.

\section{Compliance with ethical standards}

\section{Acknowledgments}

We acknowledge the help of the research Department of Mountain view Hospita, Las Vegas, Nevada.

\section{Statement of informed consent}

Informed consent was obtained from all individual participants included in the study.

\section{References}

[1] Kitabchi AE, Umpierrez GE, Miles JM, et al. Hyperglycemic crises in adult patients with diabetes: a consensus statement from the American. Diabetes Association. Diabetes Care. 2009; 32: 1335-1343.

[2] Modi A, Agrawal A, Morgan F. Euglycemic diabetic ketoacidosis. Current Diabetes Reviews. 2007; 13: $315-321$.

[3] Kabadi UM. Pancreatic ketoacidosis-ketonemia associated with acute pancreatitis. Postgraduate Med J. 1995; 71(831): 32-35.

[4] Kabadi UM. Pancreatic ketoacidosis(kabaddi syndrome) mimicking diabetic ketoacidosis. Endocrine Prac. 2015; 21(10): 1184-1185.

[5] Prater J, Chaiban JT. Euglycemic diabetic ketoacidosis with acute pancreatitis in a patient not known to have diabetes. AACE Clin Case Rep. 2015; 1(2): E88-E91

[6] Dhivahar G, Rajan P, et al. Pancreatic ketoacidosis- a rare case report. International Journal of Medical Research and Review. 2020; 8(1): 136-139. 
[7] Donowitz M et al. Glucagon secretion in acute and chronic pancreatitis. Ann Intern Med. Dec 1975; 83(6): 77881.

[8] Solomon S, et al. The glucose intolerance of acute pancreatitis: Hormonal response to arginine. Diabetes Jan 1980; 29(1): 22-26.

[9] Pillai V. A case of euglycemic pancreatic ketoacidosis- a case report. Univer J Medicine Med Special. 2018; 4(2): 13-15.

[10] Modi A et al. Euglycemic diabetic ketoacidosis- a review. Curr Diab Rev. 2017; 13(3): 315-321.

[11] Rawla P, Vellipuram AR, Bandaru SS, Pradeep Raj J. Euglycemic diabetic ketoacidosis: a diagnostic and therapeutic dilemma. Endocrinology, Diabetes \& Metabolism Case Reports. 2017; 17-0081.

[12] Prater J, Chaiban J. Euglycemic diabetic ketoacidosis with acute pancreatitis in a patient not known to have diabetes. Endocrine Practice. 1: 88-91.

[13] Savage MW, Dhatariya KK, Kilvert A, et al. Joint British Diabetes Societies. Joint British Diabetes Societies guideline for the management of diabetic ketoacidosis. Diabet Med. 2011; 28: 508-15.

[14] Umpierrez GE, Kitabchi AE. Diabetic ketoacidosis: risk factors and management strategies. Treat Endocrinol. 95108. 